[0212-7199 (2005) 22: 6; pp 288-292] ANALES DE MEDICINA INTERNA Copyright (C) 2005 ARAN EDICIONES, S.L.

An. MED. INTERNA (Madrid) Vol. 22, N. ${ }^{\circ} 6$, pp. 288-292, 2005

\title{
Tratamiento de la infección crónica por VHC en pacientes coinfectados por el VIH
}

\author{
M. L. PÉREZ GARCÍA, A. ÁLVAREZ DELGADO', A. FUERTES MARTÍN \\ Servicio de Medicina Interna II. 'Servicio de Aparato Digestivo. Hospital Universitario \\ de Salamanca. Salamanca
}

TREATMENT OF CHRONIC HEPATITIS C VIRUS INFECTION IN HUMAN INMMUNODEFICIENCY VIRUS (HIV) INFECTED PATIENTS

\section{RESUMEN}

El tratamiento de la infección por el virus de la hepatitis $\mathrm{C}$ (VHC) en los pacientes coinfectados por el virus de la inmunodeficiencia humana (VIH) se ha convertido en un tema de gran importancia, ya que las complicaciones de la hepatopatía son actualmente la primera causa de mortalidad entre el colectivo con infección por el VIH.

El objetivo de este trabajo es revisar los datos biológicos y epidemiológicos de la coinfección, y sobre todo las pautas de tratamiento a seguir, valorando las complicaciones producidas por los fármacos utilizados y sus interacciones, e informar de los resultados de los principales estudios realizados.

La pauta de tratamiento actualmente aceptada incluye interferón pegilado y ribavirina. Con estos fármacos han mejorado los resultados respecto a pautas previas, aunque la respuesta es variable según el genotipo - VHC que presente el paciente.

PALABRAS CLAVE: VHC. VIH. Coinfección HCV-VIH. Interferón. Ribavirina.

\begin{abstract}
Treatment of chronic hepatitis $C$ Virus (HCV) infection in human immunodeficiency virus (HIV) infected patients has become a topic of great importance, since the complications of chronic hepatitis are the first one cause of mortality among HIV patients.

The aim of this study is to review the biological and epidemiological data in HIV-HCV coinfection, to establish treatment guidelines taking in consideration drugs adverse effects and interations, and to report the results of the main studies carried out.

The treatment currently accepted includes pegylate interferon and ribavirin, wich have improved prior treatments, but the respouse rate depends on HCV genotype.
\end{abstract}

KEY WORDS: HCV. HIV. HCV-HIV coinfection. Interferon. Ribavirin.

Pérez García ML, Álvarez Delgado A, Fuertes Martín A. Tratamiento de la infección crónica por VHC en pacientes coinfectados por el VIH. An Med Interna (Madrid) 2005; 22: 288-292.

\section{INTRODUCCIÓN}

Hasta el año 1996 la evolución de los paciente infectados por el virus de la inmunodeficiencia humana (VIH) era hacia estadio de sida y la mayor parte acababan falleciendo en un corto espacio de tiempo. La introducción de los tratamientos antirretrovirales de gran actividad (TARGA) ha disminuido la morbimortalidad y aumentado la supervivencia, y con ello el desarrollo de enfermedades que previamente no se observaban dado su tiempo de latencia. En este grupo están las enfermedades hepáticas y sus complicaciones, producto de la infección crónica de virus hepatotropos (VHB y VHC generalmente), cuya infección esta muy ligada a la del VIH (1).
En esta revisión vamos a dedicarnos exclusivamente al virus de la hepatitis C (VHC), ya que se ha convertido en una de las principales causas de ingreso hospitalario y muerte en el grupo de pacientes con infección por el VIH $(2,3)$.

\section{BIOLOGÍA}

Tanto el VIH como el VHC son RNA virus y presentan una cinética viral muy similar. Ambos tienen una gran variabilidad genética y alta tasa de mutación, debido a que carecen de enzimas correctores de errores de replicación. La diferencia principal entre ellos es que el VIH integra el genoma en la

Trabajo aceptado: 25 de enero de 2005

Correspondencia: M. L. Pérez García. C/ Toledo, 1-3, 3 izqda. 37005 Salamanca, e-mail: marisapergar@hotmail.com 
célula infectada, mientras que el VHC no se integra y se replica en el citoplasma del hepatocito, de ahí que tras inhibición de la replicación el VHC si puede erradicarse, mientras que el VIH en su mejor respuesta puede pasar a estado crónico latente sin replicación (4).

\section{EPIDEMIOLOGÍA}

En España actualmente se calcula que hay entre 100.000 y 150.000 infectados por el VIH. Más de un $60 \%$ de estos pacientes adquirió la enfermedad por vía parenteral, y en este grupo se sabe que el $90 \%$ se encuentran coinfectados por VHC, por lo que puede calcularse que existen entre $50.000 \mathrm{y}$ 80000 pacientes con coinfección (4).

La distribución por genotipo del VHC en España se ve influida por distintos factores geográficos, la edad, el sexo, la vía de transmisión, etc., con una distribución aproximada del $65,5 \%$ genotipo $1,22,2 \%$ el $2,8,5 \%$ el 4 y en último lugar el genotipo 3 con un 2,3\%. Dentro del grupo de los UDVP existe un predominio del genotipo 1a y le siguen el 3 y el $4(5,6)$.

El riesgo de transmisión materno-fetal del VHC aumenta del 7 al $20 \%$ en pacientes coinfectados (6).

\section{HISTORIA NATURAL}

En general el VIH acelera la progresión de la infección por VHC (7). La evolución a cirrosis se produce en menos tiempo y es mayor el porcentaje de evolución a hepatocarcinoma (2$4 \%$ al año de evolución) $(8,9)$.

La tasa de progresión de fibrosis se ha visto inversamente relacionada con el recuento de linfocitos CD4+ (mayor grado si CD4+ por debajo de $200 \mathrm{cel} / \mathrm{mm}^{3}$ ) (10), presentando también mayores niveles de RNA del VHC en VIH con mal control.

Se han estudiado determinadas variables que actúan como cofactores que influyen en la aceleración de la progresión: edad (momento de la infección > 25 años), sexo (varones), obesidad, tiempo transcurrido desde la infección, ingesta de alcohol (> $50 \mathrm{~g} / \mathrm{día}$ ), otros virus hepatotropos asociados (principalmente VHB), niveles elevados de ALT, genotipo 1 del VHC ( probablemente por ser el más antiguo y que asocia mayor tiempo de infección, o mayor agresividad) y recuento de linfocitos CD4+<200 cel/ $/ \mathrm{mm}^{3}(11,12)$.

No existen evidencias claras de peor evolución del VIH en pacientes coinfectados, aunque se habla de peor recuperación de linfocitos CD4+ (13-15). Si parece clara la mayor dificultad para realizar tratamiento antirretroviral por mayor toxicidad hepática.

\section{¿A QUIÉN TRATAR?}

Desde el punto de vista del VIH, pacientes con buena situación inmunológica, con CD4+ por encima de $500 \mathrm{cel} / \mathrm{mm}^{3}$ Esta sería la condición ideal, pero si el paciente se encuentra en un rango entre 500-350 se puede individualizar y valorar la situación del paciente ( grado de fibrosis, adherencia, etc).

Si la situación lo permite es preferible tratar antes de iniciar el TARGA. Si el paciente está en tratamiento procurar pautas estable y lo menos hepatotóxicas posible.

Desde el punto de vista del VHC, los pacientes para tratar serían aquellos que tienen ALT persistentemente elevada,
PCR positiva para VHC, hepatopatía no descompensada y biopsia con fibrosis o moderada inflamación $(19,20)$.

En todas las situaciones hay que valorar contraindicaciones del tratamiento: cirrosis descompensada, UDVP activo, etilismo activo, embarazo o lactancia, enfermedades psiquiátricas descompensadas, enfermedad cardiovascular no controlada, alteraciones de función tiroidea, alteraciones hematológicas graves con anemia $(<12 \mathrm{~g} / \mathrm{dl})$, neutropenia $\left(<1.500 / \mathrm{mm}^{3}\right)$, trombopenia $\left(<90.000 / \mathrm{mm}^{3}\right)$ o insuficiencia renal (aclaramiento de creatinina $<50 \mathrm{ml} / \mathrm{h})(19)$.

El tema de la biopsia hepática es un tema controvertido. En general se dice que es conveniente, pero no imprescindible. Se debe ofrecer siempre, informando de los riesgos y los beneficios. La negativa a realizar biopsia no debe descartar tratamiento si estuviera indicado.

Es importante realizarla porque nos aporta información sobre el pronóstico de la hepatopatía informando del grado de fibrosis, identifica pacientes con cirrosis (en los cuales persiste la dudosa indicación de tratamiento), nos ayuda a descartar otras etiologías (principalmente toxicidad), y evitar tratamientos innecesarios (ausencia de fibrosis) (16).

Se debe realizar si va a influir en la decisión del tratamiento. Mayor interés en los genotipos 1 y 4 en los cuales las respuesta al tratamiento es peor y es más importante saber el estadio de la fibrosis (18).

En algunos centros se están iniciando estudios con Fibroscan, técnica que realiza mediciones de la rigidez hepática, valorando la conducción en el tejido hepático y equiparándolo a los distintos grados de fibrosis, sin necesidad de realizar biopsia hepática.

Otro tema controvertido es si debe tratarse a pacientes con transaminasas normales. En monoinfectados los resultados de los estudios recomiendan tratamiento (21), pero en los coinfectados existen más dudas. En general las transaminasas normales suele asociarse a menos grado de fibrosis (normal 14\%, mínima 34\%) (22), y predominio de genotipo 2-3 (22). En los pacientes con genotipo 2 y 3 se recomienda tratamiento dada la mejor respuesta, no siendo imprescindible la biopsia, pero en genotipos 1 y 4 por la peor respuesta es conveniente realizar biopsia hepática y valorar el grado de fibrosis: en estadio I y II esperar y en estadio III y IV iniciar tratamiento, siempre dentro de ensayos clínicos (23).

\section{FACTORES PREDICTORES DE RESPUESTA}

Se asocian a peor respuesta los genotipos 1 y 4 . El genotipo 1 es el primero en conocerse y es más difícil valorar si presenta mayor agresividad o más tiempo de evolución de la infección.

Igualmente presentan peor respuesta los pacientes con carga viral VHC por encima de $800.000 \mathrm{U} / \mathrm{ml}$ y la presencia de mayor grado de fibrosis, aunque estos pacientes tiene más necesidad de tratamiento (12).

Otros factores son: coinfección con VHB, sexo varón, edad > 40 años, mayor tiempo de evolución de la infección, obesidad, esteatosis, resistencia a la insulina, etc.

\section{EFICACIA DEL TRATAMIENTO}

Hasta llegar al tratamiento actual de la VHC se han realizado numerosos estudios y varias pautas de tratamiento (interfe- 
rón convencional en monoterapia, asociado a ribavirina, interferón pegilado en monoterapia, y asociado a ribavirina.

La asociación de interferón pegilado y ribavirina es la pauta con mejores resultados tanto en monoinfectados (VHC) como en los pacientes coinfectados.

En los resultados distribuidos por genotipos, se observa la mejor respuesta en genotipo 2 y 3 frente a los resultados en genotipo 1 y 4 . También se ha observado que la respuesta en monoinfectados con genotipos 2 y 3 es la misma tanto si la duración del tratamiento son 6 meses, como si son 12 meses, mientras que en el caso de la coinfección parece que si se obtiene un ligero aumento de respuesta si el tratamiento se prolonga a los 12 meses, atribuído a una cinética de aclaramiento viral más lenta en dichos pacientes (24), por lo cual la duración del tratamiento continúa siendo un tema discutido y en estudio.

Los principales estudios publicados sobre resultados de tratamiento de infección por VHC en coinfectados por el VIH se resumen a continuación:

—En el estudio APRICOT, se incluyeron 889 pacientes de 19 países (entre los que se encuentra España), distribuidos en tres brazos que recibieron interferón convencional (3 mill UI 3 veces/sem) con ribavirina $(800 \mathrm{mg} / \mathrm{d})$, interferón pegilado alfa-2a (180 mg) en monoterapia e interferón pegilado alfa-2a con ribavirina. Los mejores resultados se observaron en el grupo de interferón pegilado alfa-2a con ribavirina (40\%) frente a interferón convencional con ribavirina (12\%) e interferón pegilado alfa-2a en monoterapia (20\%). Persistió una mejor respuesta en genotipos 2 y $3(62 \%)$ frente a la respuesta en genotipo 1 (19\%) (25).

-El estudio ACTG A5071 incluyó 133 pacientes distribuidos en dos brazos que recibireron IFN-peg-alfa-2a y ribavirina e IFN-alfa-2a y ribavirina respectivamente. Se obtuvo una normalización de las transaminasas en un 54\% de los pacientes que recibieron IFN-peg y $44 \%$ de los pacientes que recibieron IFN (26).

-El estudio RIBAVIC, incluyó 412 pacientes distribuidos en dos brazos que recibieron interferon convencional 3 veces/semana con $800 \mathrm{mg}$ de ribavirina e interferón- pegilado $1,5 \mathrm{mg} / \mathrm{kg} / \mathrm{sem}$ con $800 \mathrm{mg}$ de ribavirina. La respuesta viral sostenida fue de $26 \%$ y $38 \%$ respectivamente (27).

-Un estudio español realizado en el H. Clínico de Barcelona (28), que incluyó 95 pacientes distribuidos en dos brazos y recibieron interferon alfa-2b (3 mill UI 3 veces/sem) y ribavirina e interferon pegilado alfa-2b (100-150 mg/sem) más ribavirina (800-1200 mg/día). La respuesta viral sostenida fue de $21 \%$ y $44 \%$ respectivamente. Persistió mejor respuesta en genotipos 2, 3 con $53 \%$, respecto a genotipos 1,4 con $38 \%$.

- Hay otros estudios en marcha con distintas pautas de tratamiento, dosis de inducción de interferon pegilado (270 $\mathrm{mg} / \mathrm{sem}$ durante las 4 primeras semanas y posteriormente 180 $\mathrm{mg} / \mathrm{sem}$ ), dosis más altas de RBV (1.000 y 1.200 mg según el peso), etc.

\section{CONSIDERACIONES ESPECIALES EN ESTE GRUPO DE PACIENTES}

Tras valorar la peor respuesta al tratamiento en pacientes coinfectados, se han analizados los grupos, observando que en estos pacientes se produce una mayor tasa de suspensión del tratamiento $(30 \%)$ que en los pacientes que no padecen infección por el VIH, tanto por abandono, como por suspensión por efectos secundarios.
Siempre es necesario tener en cuenta las interacciones con el tratamiento antirretroviral.

Es probable que exista una influencia de la inmunosupresión en la respuesta (peor respuesta en pacientes con menos de 200 CD4+), otros factores asociados a peor respuesta pueden ser un mayor porcentaje de varones o el presentar mayor grado de fibrosis en la biopsia y alta viremia.

Hay varios estudios dirigidos a estudiar la dinámica de aclaramiento viral, con resultados que demuestran un aclaramiento más lento en coinfectados $(24,29,30)$. Se observan distintas fases en el aclaración con descenso rápido en la primera fase (48 h) y descenso más lento posteriormente. Estos estudios hacen pensar que en los genotipos 2 y 3 se observa un aumento de respuesta si el tratamiento se prolonga a 12 meses en vez de 6 meses como se venía realizando, pero aún no hay resultados concluyentes que permitan establecer estas pautas de tratamiento. Otros estudios sugieren que el estado de activación inmunitaria aumentada que induce el VIH puede explicar la respuesta reducida al tratamiento del VHC en pacientes coinfectados, ya que se ha observado en la expresión génica de las células mononucleares en sangre periférica, un mayor grado de activación inmunitaria en los paciente no respondedores (31).

Por otra parte en pacientes coinfectados se observa una mayor tasa de recidiva.

\section{RECOMENDACIONES}

Con los resultados obtenidos hasta la actualidad, las pautas de tratamiento recomendados deben incluir interferón pegilado asociado a ribavirina.

Interferón Pegilado: Pegintron ${ }^{\circledR}$ (IFN alfa -2b) a dosis de $1.5 \mu \mathrm{g} / \mathrm{kg} / \mathrm{semana}$ o Pegasys ${ }^{\circledR}$ ( IFN alfa -2a) a dosis de 180 $\mu \mathrm{g} / \mathrm{semana}$.

Ribavirina: en genotipo 2 y 3 tratar con $800 \mathrm{mg} /$ día y en genotipo 1 y 4 dosis dependiente del peso del paciente $(<75$ kg $1.000 \mathrm{mg}$ y > $75 \mathrm{~kg} 1.200 \mathrm{mg}$ ) (32).

En relación a la duración del tratamiento, hasta ahora los genotipos 2 y 3 venían realizando 6 meses de tratamiento y los genotipos 1 y 412 meses, pero en la actualidad existen dudas y dado los resultados de los últimos estudios probablemente se varíen las recomendaciones aumentando la duración del tratamiento a 12 y 18 meses respectivamente, aunque aún no existen resultados concluyentes.

\section{VALORACIÓN DE LA RESPUESTA AL TRATAMIENTO}

La valoración de la respuesta al tratamiento se realiza con una determinación de la carga viral a la 12 semana de tratamiento, y se considera respuesta virológica precoz (RVP) el descenso de la carga de 2 log o niveles indetectables de RNA. Este parámetro es predictivo de respuesta sostenida.

Si no existe respuesta virológica precoz se debe suspender el tratamiento. Igualmente si a los 6 meses de tratamiento la PCR persiste positiva.

Sólo en pacientes en los que se observa una curva con cinética viral descendente, sin llegar a descenso de 2 log, con condiciones inmunológicas adecuadas, sin complicaciones y con buena tolerancia se puede independizar y continuar el tratamiento. 
Se considera respuesta viral sostenida (RVS), si la PCR del VHC persiste negativa a los 6 meses post-tratamiento.

\section{EFECTOS SECUNDARIOS}

Es importante tener en cuenta que en los pacientes infectados por el VIH existen muchas contraindicaciones para el tratamiento y entre los pacientes candidatos a él, un alto porcentaje lo suspenden. Este abandono suele estar causado fundamentalmente por los efectos secundarios del interferón (60-70\%).

El interferón produce cefalea, síndrome pseudogripal, irritabilidad, insomnio, alteraciones psiquiátricas, neutropenia, reacciones locales, etc. (32)

En nuestros paciente el control de la neutropenia debe ser fundamental, sobre todo en los primeros meses de tratamiento y si los valores de neutrófilos descienden al rango de $750 / \mathrm{mm}^{3}$ ajustar la dosis, disminuyendo a $135 \mu \mathrm{g} / \mathrm{kg} / \mathrm{semana}$ o incluso a $90 \mu \mathrm{g} / \mathrm{kg} / \mathrm{semana}$ (en el caso del Interferon alfa-2a) y si alcanzara niveles alrededor de $500 / \mathrm{mm}^{3}$ suspender el tratamiento. Puede manejarse la neutropenia con factores estimulantes de colonias e intentar mantener el interferón a dosis plenas.

En el caso de la trombopenia, cuando alcanzan valores entorno a las $50.000 / \mathrm{mm}^{3}$ se ajusta a dosis de $90 \mu \mathrm{g} / \mathrm{kg} / \mathrm{sema}-$ na y la suspensión si los valores se encuentran por debajo de $25.000 / \mathrm{mm}^{3}$.

Son efectos secundarios frecuentes en el tratamiento con ribavirina, la anemia, anorexia, disnea, prurito, rash, sequedad piel, alopecia, cefalea, mialgias, insomnio, etc.

\section{Bibliografía}

1. Bica I, McGovern B, Dhar R, Stone D, McGowan K, Scheib R, et al. Increasing mortality due to end-stage liver disease in patients with human immunodeficiency virus infection. Clin Infect Dis 2001; 32: 492-7.

2. Monga HK, Rodriguez-Barradas MC, Breaux K, Khattak K, Troisi CL, Vélez $\mathrm{M}$, et al. Hepatitis $\mathrm{C}$ virus infection-related morbidity and mortality among patients with human immunodeficiency virus infection. Clin Infect Dis.2001; 33:240-7.

3. Laguno M, Schez-Tapias JM, Murilla J, Foros X, Blanco J.L. Martinez $\mathrm{E}$, et al. Advances in the diagnosis and treatment of Hepatitis $\mathrm{C}$ Virus infection in patients with and without HIV infection. Enferm Infecc Microbiol Clin 2005; 23 (1): 32-40.

4. Ros J y Glez García J. H. La Paz. Madrid. Control Calidad SEIMC. Infección por el virus de la Hepatitis $\mathrm{C}$ en pacientes infectados por el virus de la Inmunodeficiencia Humana: características y aspectos de su epidemiología, diagnóstico y tratamiento. http://www. seimc.org/control/revi_sero/VHCVIH.htm

5. Hernández JM, Sauleda S, Massuet LI, Esteban JI, Esteban R, Guardia J. HCV genotype distribution among HCV positive blood donors and outpatients according to epidemiological features. AABB Annual Meeting. San Francisco 1999 (Abstract 304).

6. Sauleda S, Juárez A, Esteban JI. Tratamiento de la hepatitis C en pacientes coinfectados por VIH. Gastroenterol Hepatol 2002; 25: 337-41.

7. Benhamou Y, Bochet M, Di Martino V, Charlotte F, Azria F, Coutellier A, et al. Liver fibrosis progresión in human immunodeficiency virus and hepatitis C virus coinfected patients. Hepatology 1999; 30: 1054-8.

8. García-Samaniego J, Rodriguez M, Berenguer J, Rodriguez-Rosado $\mathrm{R}$, Carbo J, Asensi V et al. Hepatocellular carcinoma in HIV-infected patients with chronic hepatitis C. Am J Gastroenterology 2001; 96: 179-83.
El efecto secundario más importante en nuestros pacientes es la anemia. Se recomienda en valores de $\mathrm{Hb}<10 \mathrm{~g} / \mathrm{dl}$ bajar dosis a $600 \mathrm{mg}$ y por debajo de $8,5 \mathrm{~g} / \mathrm{dl}$ supender el tratamiento. Al igual que el manejo de la neutropenia secundaria al interferón, en este caso la anemia puede corregirse con eritropoyetina intentando mantener la dosis adecuada de ribavirina.

\section{INTERACCIONES}

El interferón no presenta interacciones significativas con el tratamiento antirretroviral, salvo la posibilidad de trastornos psiquiátricos si el paciente se encuentra en tratamiento con efavirenz.

En el caso de la ribavirina si existe interacciones importantes. La asociación de zidovudina (AZT) puede aumentar el riesgo de anemia que ya produce la ribavirina por si sóla. En el caso de la didanosina (ddI) aumenta el riesgo de pancreatitis aguda. En la asociación de didanosina y estavudina (d4T) el riesgo de desarrollar acidosis láctica es muy alto (33). Si se asocian varios análogos como por ejemplo la estavudina y la didanosina, la lipodistrofia puede aumentar y también con esta asociación existe más riesgo de descompensación hepática.

Las recomendaciones reflejan no usar nunca d4T, con ddI y ribavirina dada la toxicidad mitocondrial que produce dicha asociación (hay descritos casos de muertes) (34). Evitar asociar ddI y ribavirina. Vigilar si se asocia d4T y ribavirina.

El uso de nevirapina o ritonavir a altas dosis aumentan el riesgo de hepatotoxicidad (35).
9. Puoti M, Bruno R, Soriano V, Donato F, Gaeta GB, Quizan GP, et al. Hepatocellular carcinoma in HIV- infected patients: epidemiological features, clinical presentation and outcome. AIDS 2004; 8 (17): 2285-93.

10. Thomas DL, Astemborski J, Rai RM, Anania FA, Schaeffer M, Galai N et al. The natural history of hepatitis $\mathrm{C}$ virus infection: host, viral and environmental factors. JAMA 2000; 284: 450-6.

11. Puoti M, Bonacin M, Spinetti A, Putzolu V, Govindarajan S, Zaltron S, et al. Liver fibrosis progression is related to CD4 depletion in patients coinfected with hepatitis C Virus and Human Immonodeficiency Virus. J Infect Dis 2001; 183: 134-7.

12. Martin Carbonero L, Benhamou Y, Ponti M, Berenguer J, Mallolas J, Quereda C, et al. Incidence and predictors of sever liver fibrosis in human immunodeficiency virus-infected patients with chronic hepatitis C: a European collaborative study. Clin Infect Dis 2004; 38 (1): 128-33.

13. Piroth L, Duong M, Quantin C, Abrahamowics M, Michardiere R, Aho $\mathrm{LS}$, et al. Does hepatitis $\mathrm{C}$ virus coinfection accelerate clinical and immunological evolution of HIV-infected patients?. AIDS 1998; 12: 381-8.

14. Piroth L, Grappin M, Cuzin L, Mouton Y, Bouchard O, Raffi F, et al. Hepatitis $\mathrm{C}$ virus co-infection is a negative prognostic factor for clinical evolution in human immunodefiency virus-positive patients. J.Viral Hepat 2000; 7: 502-8.

15. Sulkowski MS, Moore R, Metha SH, Chaisson RE, Thomas D. Hepatitis C and progression of HIV disease. JAMA 2002; 228: 199-206.

16. National Institutes Health Consensus Development Conference Statemente: Manegement of Hepatitis C: 2002- June 10-12, 2002. Hepatology 2002; 36: S3-S20.

17. Quereda C, Moreno S, Moreno L, Moreno A, García-San Miguel L, Pérez-Elías MJ, et al. The role of Liver Biopsy in the Manegement of Chronic Hepatitis $\mathrm{C}$ in Patients Infected with the Human Immunodeficiency Virus. Human Pathology 2004; 35: 1083-7. 
18. Puoti M, Bruno R, Castelli F, Filice G, Carosi G. Liver biopsy in HIVinfected patients with chronic hepatitis $\mathrm{C}$ : pros and cons. HIV Clin Trials 2002; 3: 419-20

19. Soriano V, Puoti M, Sulkowski M, Mauss S, Cacoub P, Cargnel P, et al. Care of patients with chronic Hepatitis C and HIV co-infection: recommendations from the HIV-HCV International Panel. AIDS 2004; 18: 112.

20. González-García J, Guerra L, Amela C, del Amo J, Bruguera M, Castilla J, et al. Coinfección por VIH y virus de las Hepatitis A, B y C en adultos. Revisiones y recomendaciones de documentos de consenso de GESIDA/PNS sobre la terapia antirretroviral y enfermedades asociadas al VIH (2000-2002). Documentos. Recomendaciones. http//: www.gesidaseimc.com.

21. Zeuzem S, Diago M, Gane E., Reddy R, Pockros P, Farci P, et al. International, multicenter, rabdomized, controlled study for the treatment of patients with chronic hepatitis $\mathrm{C}$ and persistently normal ALT levels with Peginterferon alfa-2a and Ribavirin. 54th Annual Meeting of th American Association for the Study of Liver Diseases. Boston 2003. (Abstract 106)

22. Puoti C, Castellacci R, Martagnesse F, Zaltron S, Stomaiuolo G, Berga$\mathrm{mi} \mathrm{N}$, et al. Histological and virological features and follow-up of Hepatitis $\mathrm{C}$ virus carries with normal aminotransferase levels: the Italian prospective study of de asymptomatic C carries. J Hepatol Jul 2002. 37 (1): 117-23.

23. Diago M. ALT normales en pacientes con infección crónica por el virus de la hepatitis C: comprender la necesidad del tratamiento y optimizar el manejo del paciente. Gastroenterología Práctica. Número Especial. Congreso de la Asociación Española para el estudio del higado. Feb2004.2-5.

24. Soriano V, Núñez M, Camino N, Maida I, Barreiro P, Romero M, et al. Hepatitis C virus - RNA clearance in HIV- coinfection patients with chronic hepatitis $\mathrm{C}$ treated with pegylated interferon plus ribavirin. Antivir Ther 2004; 9: 505-9.

25. Torriani F, Rodriguez-Torres M, Rockstroh JK, Lissen E, Glez-García J, Lazzarin A, et al. For the APRICOT Study Group. Peginterferon Alfa-2a plus Ribavirin for Chronic Hepatitis C Virus Infection in HIVInfected Patients. N Engl J Med 2004; 351: 438-50.

26. Chung R, Andersen J, Volberding P, Robbins GK, Lin T, Sherman KE, et al. Peginterferon alfa-2a plus ribavirin versus interferon alfa-2a plus ribavirin for chronic hepatitis $\mathrm{C}$ in HIV-coinfected persons. ACTG A5071. N Engl J Med 2004; 351: 451-9.

27. Carrat F, Bani-Sadr F, Pol S, Rosenthal E, Lunel-Fabiani F, Benzekri A, et al. For de ANRS HCO2 RIBAVIC Study Team. Pegylalet Interferon alfa-2b vs Standard Interferon alfa-2b, plus Ribavirin, for Chronic Hepatitis C in HIV-Infected Patients. A Randomized Controlled Trial. JAMA 2004; 292: 2839-48.

28. Laguno M, Murillas J, Blanco JL, Martinez E, Miquel R, SánchezTapias JM, et al. Peginterferon alfa-2b plus ribavirin compared with interferon alfa-2b plus ribavirin for treatment of HIV-HCV co-infected patients. AIDS 2004; 18 (13): 27-36.

29. Sherman K, Horn Ps, Rouster SD, Peters MG, Koziel MJ, Chang R et al. Modeling HCV viral kinetic response to Peg-IFN and Ribavirin in HCV-HIV coinfected patients. 54 th Annual Meeting of the American Association for the Study of Liver Diseases. Boston. October-2003.

30. Torriani FJ, Ribeiro RM, Gilbert TL, Schrenk UM, Clauson M, Pacheco $\mathrm{DM}$ et al. Hepatitis $\mathrm{C}$ virus ( $\mathrm{HCV}$ ) and human immunodeficiency virus (HIV) dynamics during HCV treatment in HCV-HIV coinfection. J Infect Dis 2003; 188: 1498-507.

31. Lempicki RA, Yang J, Dennis G, McLaughlin M, Koratich C, Kleiner $\mathrm{D}$, et al. HCV resistance to peg-interferon/ribavirin therapy is associated with increased immune activation in $\mathrm{HIV} / \mathrm{HCV}$ co-infected patients. 11 th Conference on Retrovirus and Opportunistic Infections. CROI. San Francisco.Feb-2004 (Abstract 118LB).

32. Plosker GL, Keating GM. Peginterferón-alfa-2a ( 40 KD) más ribavirina: a review of its use in hepatitis C Virus and HIV co-infection. Drugs 2004; 64: 2823-43.

33. Lafenillade A, Hittinger G, Chapadaud S. Increased mitochondrial toxicity with ribavirin in HIV-HCV coinfection. Lancet 2001: 357: 280-1.

34. Guyader D, Poisignon Y, Cano Y, Scout L Fatal lactic acidosis in a HIV-positive treated with interferon and ribavirin for chronic hepatitis C. J Hepatol 2002; 37: 289-91.

35. Sulkowski MS, Thomas DL, Chaisson R, Moore RD. Hepatotoxicity associated with antiretroviral therapy in adults infected with human immunodeficiency virus and the role of hepatitis $\mathrm{C}$ o B virus infection. JAMA 2000; 283: 74-80. 